\title{
Two-dimensional simulation for the GaAs V-groove gate MESFET's
}

\author{
Y.J. Wang, S.S. Lu* \\ Department of Electrical Engineering, National Taiwan University, Taipei 106, Taiwan
}

Received 25 December 1997; received in revised form 21 May 1998

\begin{abstract}
V-groove gate MESFET's were investigated in this paper by 2D simulation. In these devices, an interesting draininduced-carrier-accumulation (DICA) effect was found. This effect could explain the origin of the output conductance and the dependence of the pinch-off voltages on the drain bias (i.e. short-channel effect). An analytical formula with physical meaning parameters for describing the DICA effect were also proposed. In addition, different substrate effects (including the undoped $\mathrm{GaAs}$, undoped $\mathrm{Al}_{0.3} \mathrm{Ga}_{0.7} \mathrm{As}$ and p-doped $\mathrm{GaAs}$ substrates) on the $\mathrm{V}$ groove gate MESFET's were simulated. The simulated $I-V$ characteristics of the three devices with the different substrates exhibited short channel effects but the mechanisms of the short channel effect were found different. In the device with undoped GaAs substrate, the mechanism of the short-channel effect was due to the leakage current in the substrate. However, in that with the undoped AlGaAs substrate, it was the DICA effect. As for the device with the p-doped GaAs substrate, both the leakage current and the DICA were found. (C) 1998 Elsevier Science Ltd. All rights reserved.
\end{abstract}

\section{Introduction}

2D Simulation has been widely applied in the research of semiconductor device physics. In 1970, Kennedy and O'Brien simulated GaAs FET [1]. Reiser investigated the substrate leakage in the GaAs MESFET [2]. In 1976, Yamaguchi et al. discussed the stability of the dipole domain in the GaAs MESFET [3]. However, the V-groove gate GaAs FET has not been simulated up to now. The V-groove GaAs MESFET was first fabricated by Kohn [4] in 1975. It had a higher cut-off frequency $\left(f_{\mathrm{t}}\right)$ than those of the traditional MESFET with planar gate. The potential in microwave applications has also been shown [5]. Hence, in this paper, the V-groove MESFET was simulated for further understanding.

Substrate effects in the GaAs FET's with planar gate have been studied both analytically (Shur and Eastman

\footnotetext{
* Corresponding author. Tel.: +886-2-2363-5251; Fax: +886-2-2363-8247; E-mail: sslu@cc.ee.ntu.edu.tw
}

for GaAs MESFET's [6]) and by 2D numerical simulation (Reiser for GaAs JFET's [2]). In these studies, it was found that the substrate would cause the short channel effect in the device because the current could leak into the substrate. The leakage current would become larger when the gate length became shorter. Hence, it caused the finite output resistance of the device and the increase of the pinch-off voltage $\left(\left|V_{\mathrm{p}}\right|\right)$ with the increase of $V_{\mathrm{ds}}$. In the paper, we will discuss how different substrates affect the mechanisms of the short-channel effect in the V-groove gate MESFET's.

\section{Device structure}

The device structure of the V-groove gate MESFET can be understood by its fabricating processes, which had the following steps. First, we had (100) GaAs wafers with an n-type epitaxial layer, $907 \mathrm{~nm}$ thick and $1 \times 10^{17} / \mathrm{cm}^{3}$ doped, grown on the substrates with 800 $\mathrm{nm}$ thick buffer layer. Three kinds of buffer layer were chosen: (a) undoped GaAs, (b) undoped $\mathrm{Al}_{0.3} \mathrm{Ga}_{0.7} \mathrm{As}$ 


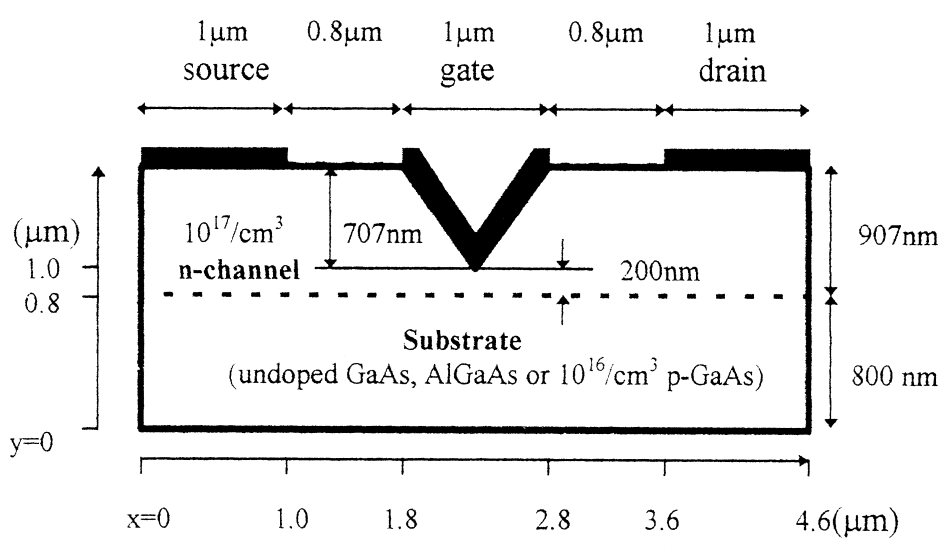

Fig. 1. Cross-sectional view of the V-groove MESFET's.

or (c) $10^{16} / \mathrm{cm}^{3}$ p-type GaAs. Second, the wafers were etched to form the device mesa and then the ohmic contacts for drain and source were evaporated. Third, we aligned the $1 \mu \mathrm{m}$ gate mask along the (011) direction and then etched the gate anisotropically to form a
V-groove. For example, $\mathrm{H}_{2} \mathrm{SO}_{4}-\mathrm{H}_{2} \mathrm{O}_{2}-\mathrm{H}_{2} \mathrm{O}$ (1:8:1) is such an anisotropic etching solution for GaAs. This solution has a slower etching rate on the (111) planes than that on the (100) planes (etching rate: $\operatorname{GaAs}(111): \operatorname{GaAs}(100)=0.24: 1)$ [7]. Therefore, the

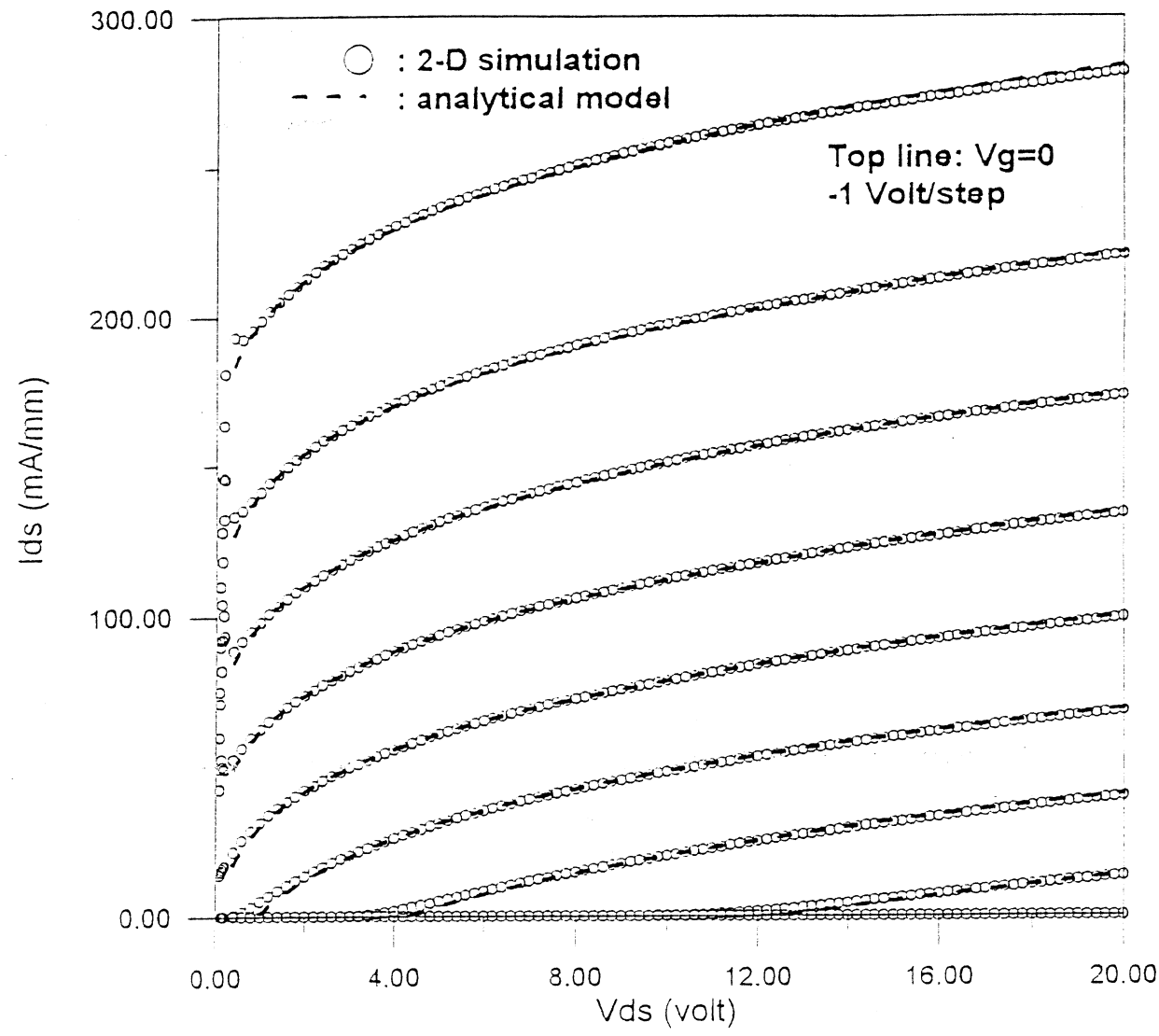

Fig. 2. $I-V$ characteristics of the $\mathrm{V}$-groove MESFET neglecting the substrate. 

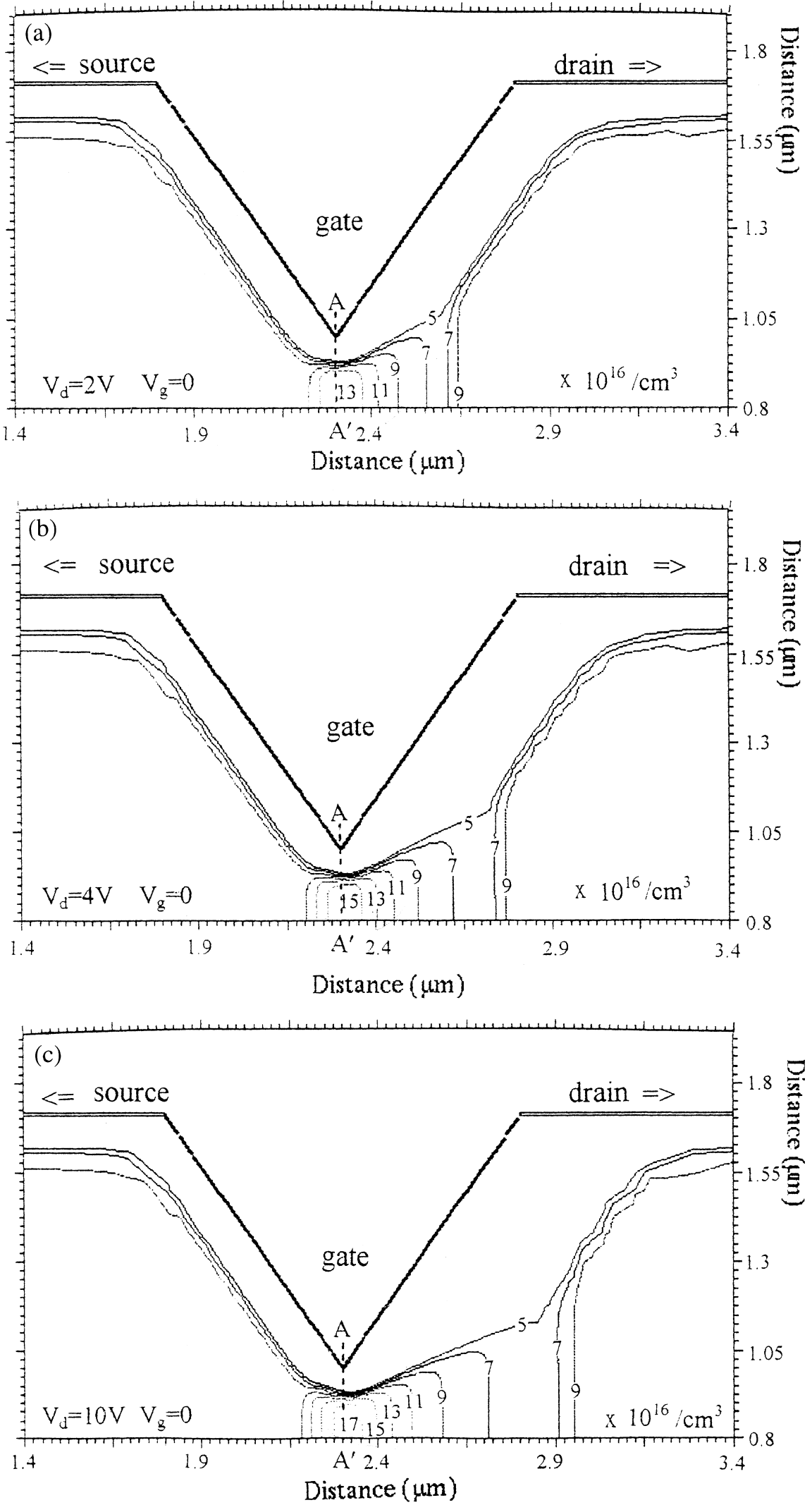

Fig. 3. Electron concentration of the V-groove MESFET neglecting the substrate (a) $V_{\mathrm{d}}=2 \mathrm{~V}, V_{\mathrm{g}}=0 \mathrm{~V}$; (b) $V_{\mathrm{d}}=4 \mathrm{~V}, V_{\mathrm{g}}=0 \mathrm{~V}$; (c) $V_{\mathrm{d}}=10 \mathrm{~V}, V_{\mathrm{g}}=0 \mathrm{~V}$. 
etched V-groove had the (111) planes as its two side walls. As a result, the ratio of its width of the Vgroove to its depth was $\sqrt{2}: 1$. Since the V-groove had $1 \mu \mathrm{m}$ top opening, its depth was $707 \mathrm{~nm}$ and the resulting channel thickness became $200 \mathrm{~nm}$. Finally, Schottky metals were evaporated onto the groove as gate electrode. They formed a $0.9 \mathrm{eV}$ high Schottky, barrier to control the conduction of the channel. Thus, the V-groove MESFET was fabricated. Its cross-sectional view is shown in Fig. 1.

\section{Simulation methods}

The simulation is by way of the finite-difference method. This method divides the device with many nodes. At each node, it solves the Poisson equation and continuity equations accompanied with given boundary conditions simultaneously to get its potential and carrier concentration. The smallest spacing (about
$7 \mathrm{~nm}$ ) between two nodes is located under the Vgroove tip in the channel. Then the spacing becomes larger in the ratio of about 1.07 toward source, drain, substrate bottom and the upper electrodes. Also, in the regions where the physical quantities do not change very much between two neighbor nodes, fewer nodes were used to reduce the total number of nodes and, as a result, the simulation time was reduced.

The velocity v.s. electric field model with negative differential mobility slope was used to simulate the electron behavior in the channel. Its formula is as follows:

$$
v=\frac{\mu E+v_{\mathrm{s}}\left(E / E_{0}\right)^{4}}{1+\left(E / E_{0}\right)^{4}}(\mathrm{~cm} / \mathrm{s})
$$

where $\mu$ is the low-field mobility, $v_{\mathrm{s}}$ is the saturation velocity $\left(7.8 \times 10^{6} \mathrm{~cm} / \mathrm{s}\right)$ and $E_{0}$ is the characteristic Efield $(3000 \mathrm{~V} / \mathrm{cm})$. This formula was derived from intervalley scattering by Butcher and Fawcett in

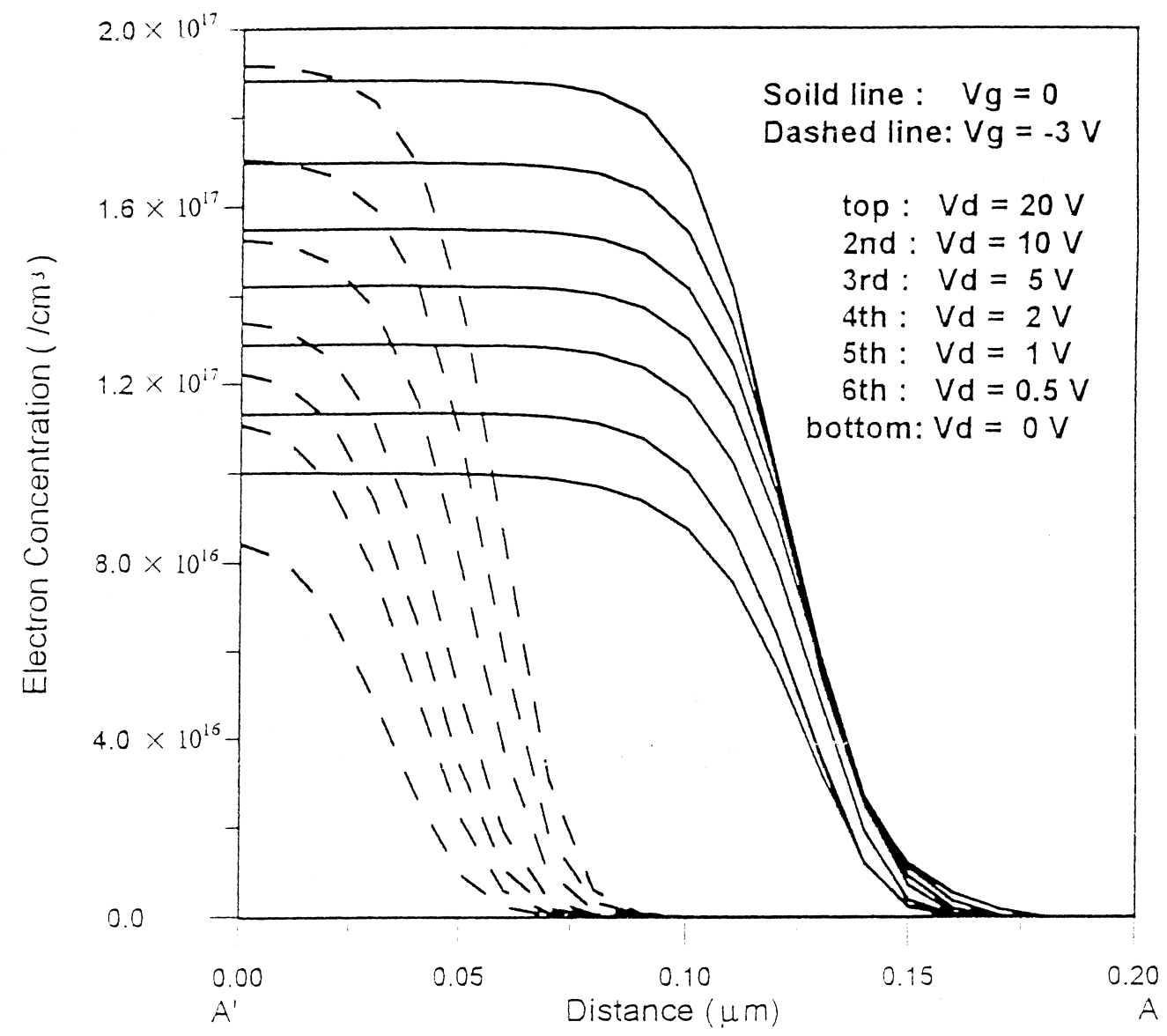

Fig. 4. Electron concentration under the tip of the V-groove gate neglecting the substrate. 


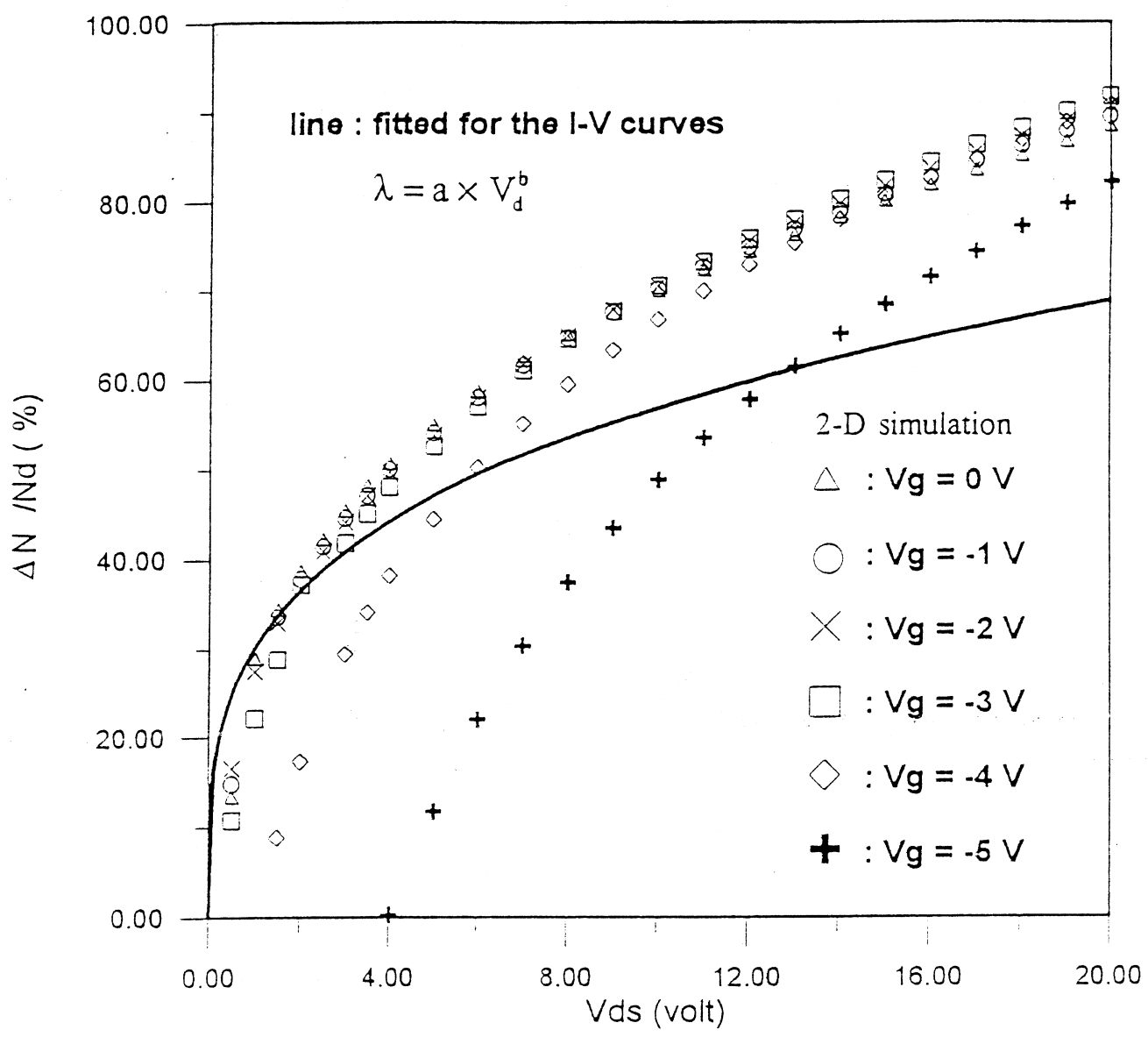

Fig. 5. Extra electron percentage under the tip of the V-groove gate and $\lambda$ from analytical model v.s. $V_{\mathrm{d}}$.

1966 [8] and evidenced in 1967 by Ruth and Kino in an experiment [9] and was referred to by Copeland (1967) [10], Thim (1968) [11], Barnes (19741976) $[12,13]$ and Yamaguchi (1976) [3]. It was believed to be the most accurate model for the highfield mobility of GaAs. The non-stationary hot electron effect was not taken into consideration for simplicity, because it could always be included in the increase of the saturation velocity. Also, contact resistances of drain and source were neglected. The gate Schottky barrier was assumed to be $0.9 \mathrm{eV}$. Surface depletion is modeled by a fix charge in the surface $\left(-1 \times 10^{12} \mathrm{e} / \mathrm{cm}^{2}\right)$ and hence a $0.6 \mathrm{~V}$ to high surface potential was built. The boundary condition except on the electrodes is the Neumann boundary condition: $\vec{n} \cdot \nabla \varphi=0$, where $\vec{n}$ is the normal unit vector at boundary; $\varphi$ is potential. The boundary condition on $\mathrm{GaAs} / \mathrm{AlGaAs}$ heterojunction is that $\overrightarrow{\mathrm{n}} \cdot \varepsilon_{\mathrm{GaAs}} \nabla \varphi_{\mathrm{GaAs}}-\overrightarrow{\mathrm{n}} \cdot \varepsilon_{\mathrm{AlGaAs}} \nabla \varphi_{\mathrm{AlGaAs}}=\sigma_{\mathrm{s}}$, where $\varepsilon$ is the permittivity and $\sigma_{\mathrm{s}}$ is the interface charge density.

\section{Simulation results}

\subsection{Substrate neglected}

The simulation results of the $I-V$ characteristics of the V-groove MESFET with the substrate neglected are shown in Fig. 2. We can see that the device exhibits a short-channel effect obviously because its $I_{\mathrm{d}}$ increases with $V_{\mathrm{d}}$ and $\left|V_{\mathrm{p}}\right|$ increases with $V_{\mathrm{d}}$. The mechanism of the short-channel effect can not be due to the leakage current in the substrate (since we did not take the substrate into account). Instead, we found that a new mechanism: drain-induced-carrier-accumulation (DICA) effect was the reason to cause this phenomena.

The DICA effect is different from the short-channel effect in MESFETs with submicron planar gate. The short-channel effect in MESFETs with submicron planar gate is resulted from the poor aspect ratio (gate length/channel thickness) of the devices. However, since there is no well-defined gate length for the V- 

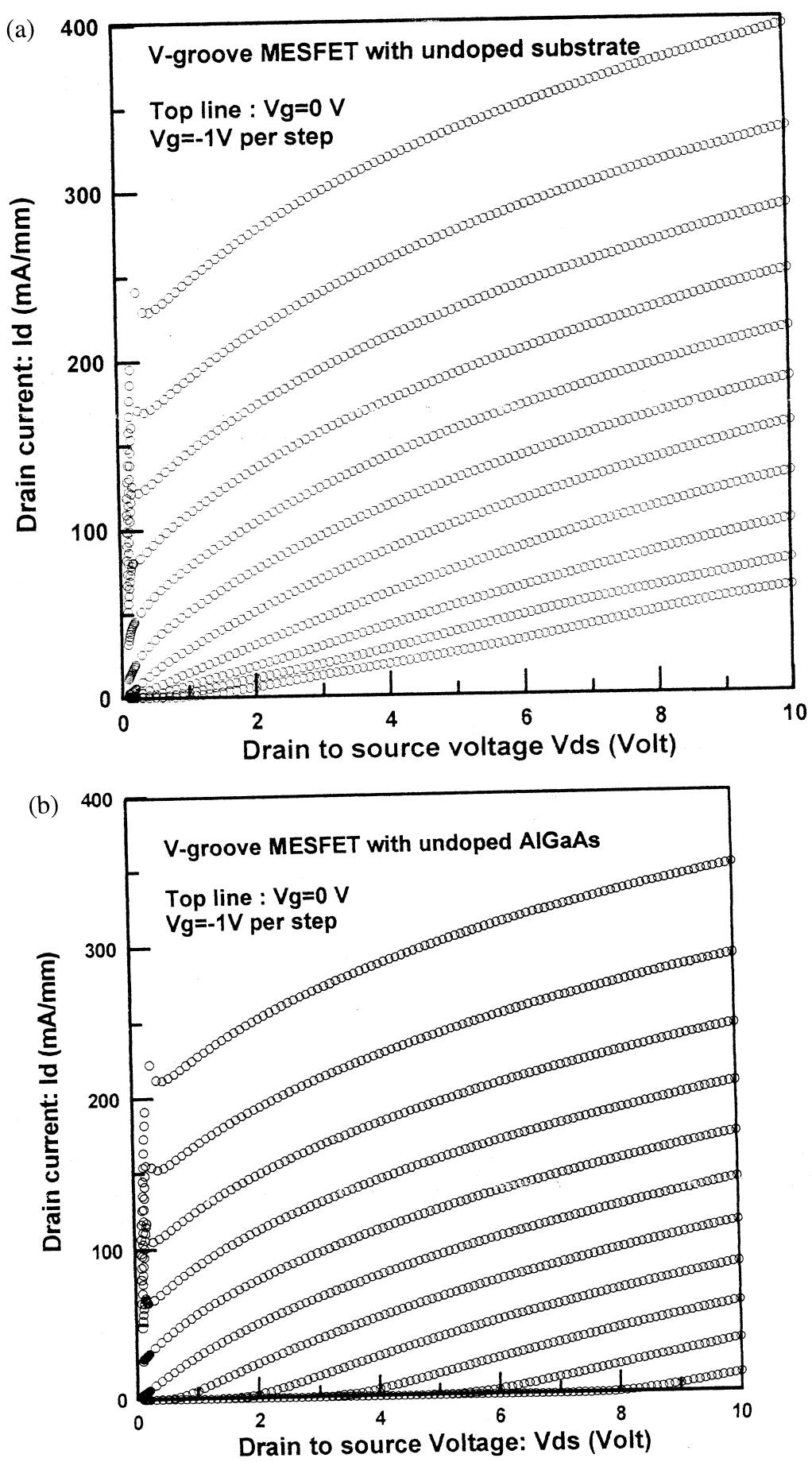

Fig. 6. $I-V$ characteristics of the V-groove MESFET's with (a) undoped GaAs, (b) undoped $\mathrm{Al}_{0.3} \mathrm{Ga}_{0.7} \mathrm{As}$ and (c) $10^{16} / \mathrm{cm}^{3}$ p-type GaAs substrate. 


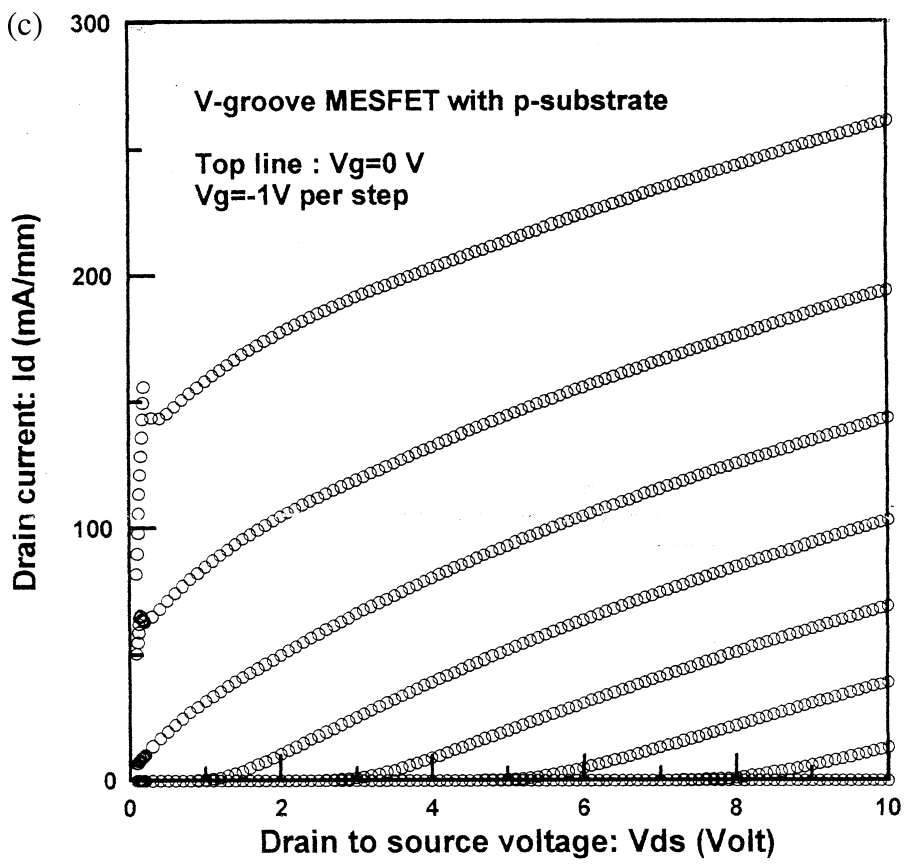

Fig. 6 (continued)

groove gate MESFET, there is no well-defined aspect ratio either.

The DICA effect is resulted from the increase of the accumulated carrier under the $\mathrm{V}$ groove gate due to increasing $V_{\mathrm{d}}$. It is well known that the negative differential mobility of GaAs causes the dipole domain under gate in the GaAs MESFET with planar gate [3]. However, in the V-groove gate MESFET, the electron accumulation region of the dipole domain is just under the tip of V-groove. Hence, the extra electron caused the extra channel current. When $V_{\mathrm{d}}$ became larger, the dipole became stronger. Then, the electron accumulated under the tip of the $\mathrm{V}$-groove gate became larger. As the result, the $I_{\mathrm{d}}$ increased with $V_{\mathrm{d}}$.

Fig. 3(a)-(c) show the electron distribution in the Vgroove gate MESFET at $V_{\mathrm{d}}=2,4$ and $10 \mathrm{~V}$ and $V_{\mathrm{g}}=0$, respectively. We can see that the electron accumulation of the dipole domain for various $V_{\mathrm{d}}$ 's is just under the tip of the V-groove because it is the narrowest position. Furthermore, if we sketch the electron distribution at different $V_{\mathrm{d}}$ 's along the line (i.e. A-A' in Fig. 3) from the V-groove's tip to the bottom of the channel as in Fig. 4, we can see clearly that the number of accumulated electrons under the gate is getting larger as $V_{\mathrm{d}}$ increases. Fig. 5 shows that the percentage increase $\left(\Delta N / N_{\mathrm{d}}\right)$ of the extra electron maintains almost the same value at different $V_{\mathrm{g}}$ 's until it drops when $V_{\mathrm{g}}$ approaches $V_{\mathrm{p}}$. At this point, the increase of $\Delta N / N_{\mathrm{d}}$ is independent of $V_{\mathrm{g}}$.
Furthermore, the $I-V$ curves in Fig. 2 could be described by an analytical model, which is modification from the velocity saturation model for GaAs MESFET's with four extra parameters as follows:

$$
I_{\mathrm{d}}=q \times N_{\mathrm{d}} \times(t-w) \times v_{\text {sat }} \times(1+\lambda)
$$

and

$$
W=\sqrt{\frac{\alpha \varepsilon\left(V_{\mathrm{g}}+V_{\mathrm{bi}}\right)}{q N_{\mathrm{d}}}}
$$

where $\lambda=a \times V_{\mathrm{d}}^{\mathrm{b}}, \quad \alpha=c \times(1+k \lambda)^{-1}$ and $q$ represents unit charge $\left(1.6 \times 10^{-19} \mathrm{C}\right) ; N_{\mathrm{d}}$, channel doping $\left(10^{17} / \mathrm{cm}^{3}\right) ; t$, channel thickness $(0.2 \mu \mathrm{m}) ; V_{\text {sat }}$, saturation velocity $\left(7.8 \times 10^{6} \mathrm{~cm} / \mathrm{s}\right) ; w$, depletion width; $\varepsilon$, the permittivity of $\operatorname{GaAs}\left(13.1 \varepsilon_{0}\right)$ and $V_{\mathrm{bi}}$, the built-in potential $(0.9 \mathrm{~V})$. Here four parameters $a, b, c$ and $k$ were determined by curve fitting. Through the above equations, we can fit the $I-V$ curves very well as also shown in Fig. 2. The values of $a, b$ and $c$ obtained are $0.303,0.274$ and 1.386 , respectively. $k$ is 1.622 .

The meaning of the first two equations can be readily interpreted by the DICA effect. The last two equations have less physical meaning. $\lambda=a \times V_{\mathrm{d}}^{\mathrm{b}}$ represents how $\left(\Delta N / N_{\mathrm{d}}\right)$ change with $V_{\mathrm{d}}$ in a simple power function as also plotted in Fig. 5. $\alpha=c \times(1+k \lambda)^{-1}$ represents the depletion width slightly affected by the electron accumulation. 


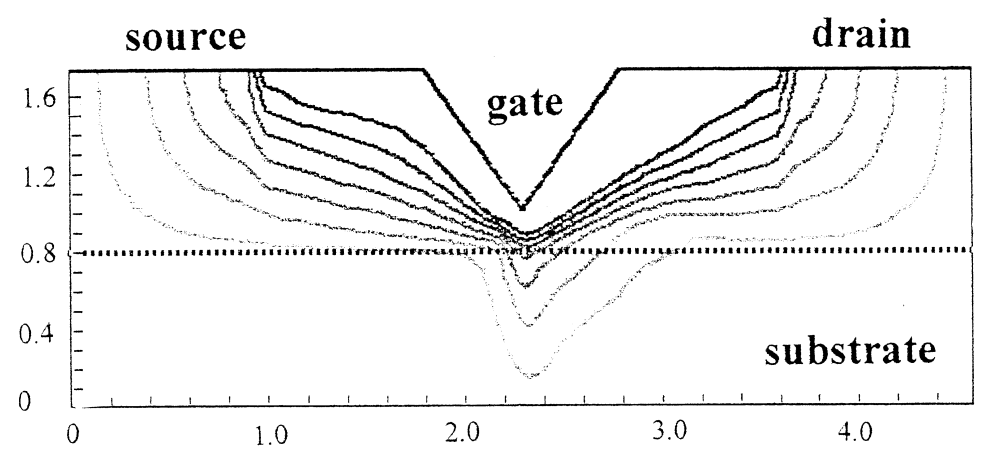

Fig. 7. Current distribution of the V-groove MESFET with undoped GaAs substrate at $V_{\mathrm{d}}=10 \mathrm{~V}$ and $V_{\mathrm{g}}=0$.

\subsection{Consideration of substrate}

The $I-V$ characteristics of the V-groove MESFET with undoped $\mathrm{GaAs}$, undoped $\mathrm{Al}_{0.3} \mathrm{Ga}_{0.7} \mathrm{As}$ and $10^{16}$ / $\mathrm{cm}^{3}$ p-type GaAs substrate are shown in Fig. 6(a), (b) and (c), respectively. We can see obviously in Fig. 6 that all of the V-groove MESFET's exhibit short-channel effect because the pinch-off voltage of the V-groove MESFET increases negatively as drain biases increase, and that the slope of the $I-V$ curve, the output conductance is very large. However, the mechanisms of the short channel effect in the three devices differ from one another. The differences will be clarified as follows.

\subsubsection{V-Groove MESFET with undoped GaAs substrate}

Like the planar gate MESFET, the short channel effect in the V-groove MESFET with undoped GaAs substrate is basically resulted from the current injection to the semi-insulating substrate. For example, it can be easily seen in Fig. 7, which exhibits the current distribution of the device at $V_{\mathrm{d}}=1.0 \mathrm{~V}$ and $V_{\mathrm{g}}=0 \mathrm{~V}$ (the 7 lines in Fig. 7 divides the device into 8 parts. this means that $1 / 8$ of the total current flows through each part) that half current is through the substrate. Furthermore, if we divide the current into two parts, in the channel and in the substrate as shown in Fig. 8(a) and (b), we can see that although its total $I_{-}$ $V$ curve exhibits short channel effect, the channel current itself does not exhibit the short channel effect. The $V_{\mathrm{p}}$ in the channel is a little less than $-5 \mathrm{~V}$. This is consistent with the fact that the leakage current almost keeps the same at different $V_{\mathrm{g}}$ 's until it is controlled by the gate when $V_{\mathrm{g}}$ drops below $-5 \mathrm{~V}$. Therefore, we concluded that the short channel effect of the total current comes from the substrate leakage.

\subsubsection{V-Groove MESFET with undoped AlGaAs-buffer layer}

The mechanism of the short channel effect in the Vgroove MESFET with undoped $\mathrm{Al}_{0.3} \mathrm{Ga}_{0.7} \mathrm{As}$ buffer was different from that in the device with undoped GaAs substrate. It was not due to the substrate leakage because the leakage current is prevented by the high bandgap material $\mathrm{Al}_{0.3} \mathrm{Ga}_{0.7} \mathrm{As}$. Instead, we found that it is due to DICA effect.

Through the above analytical equations, we can fit the $I-V$ curves of the $\mathrm{V}$-groove MESFET very well as shown in Fig. 9. The values of $a, b$ and $c$ we used are $0.390,0.391$ and 1.567 , respectively, and $k$ is 2.365 .

\subsubsection{V-Groove MESFET with p-buffer layer}

The simulation result of the V-groove MESFET with $10^{16} / \mathrm{cm}^{3}$ p-doped GaAs layer is shown in Fig. 6(c). In Fig. 6(c), we can see that the $I_{\mathrm{dss}}$ is smaller than those of the above two devices. The reason is that the $\mathrm{p}-\mathrm{n}$ junction between the $\mathrm{n}$-channel and the $\mathrm{p}$-substrate build a depletion region which reduce the channel thickness. For the same reason, the $\left|V_{\mathrm{p}}\right|$ is also smaller. Although we expect that the p-buffer will eliminate the substrate leakage current, the total current still has some portion through the substrate, especially at large drain voltage as shown in Fig. 10(a) and (b), which exhibit the channel current and the substrate leakage current, respectively. Besides, in Fig. 10(a), we can see that the channel current, unlike the one with the undoped buffer, still suffers from the short channel effect in small $V_{\mathrm{d}}$. We will show that it is because of the DICA effect. Therefore, we see the substrate leakage at large $V_{\mathrm{d}}$ and the DICA effect at small $V_{\mathrm{d}}$ in the V-groove MESFET with $10^{16} / \mathrm{cm}^{3}$ p-doped GaAs layer.

\section{Discussion}

For further investigation we would like to find out the reason why the devices with different substrate had the different mechanisms of the short channel effect. We found that it was related to the electron behavior closely under the V-groove tip. 

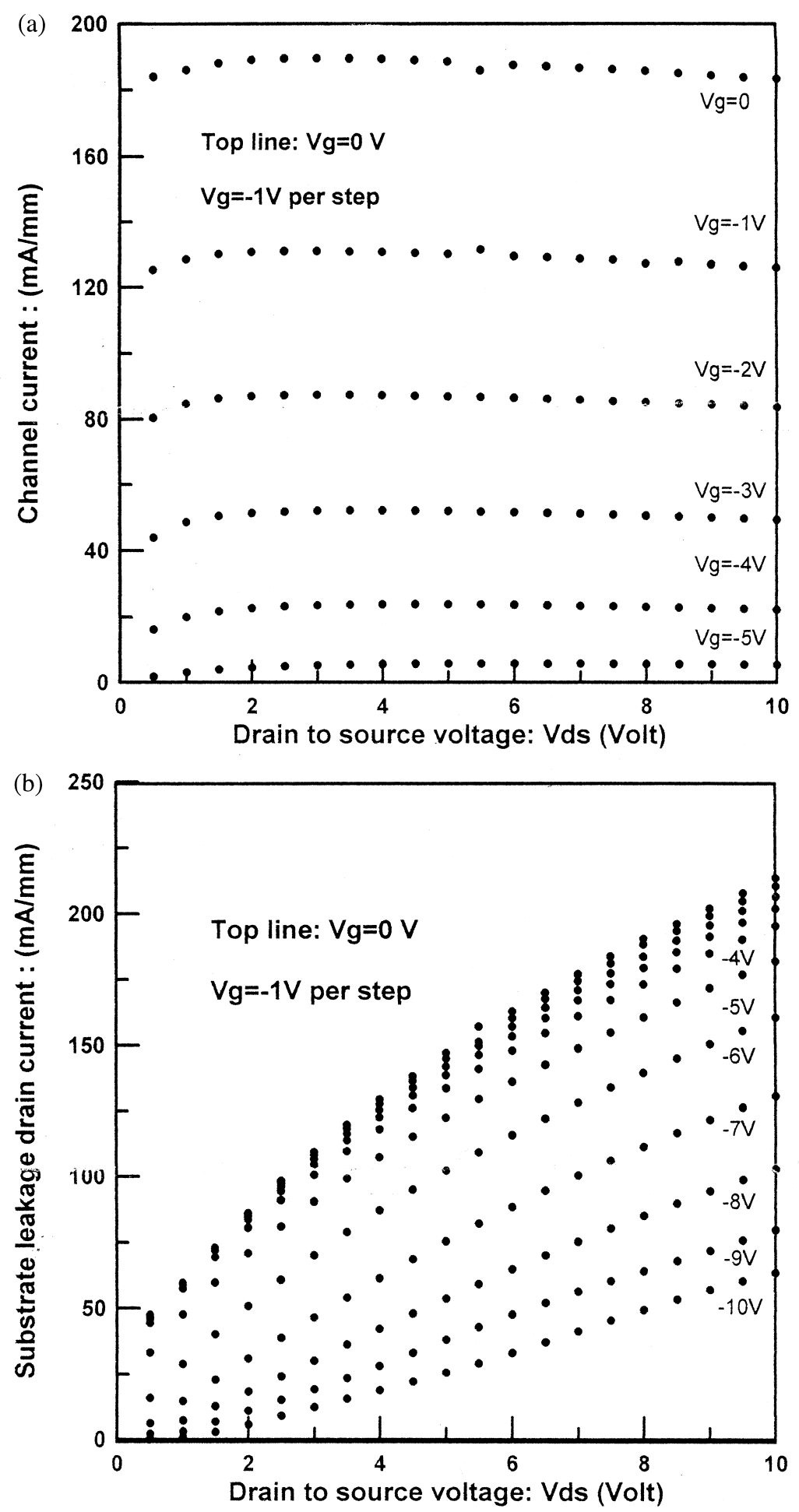

Fig. 8. (a) Channel current and (b) substrate leakage current of the V-groove MESFET with undoped GaAs substrate. 


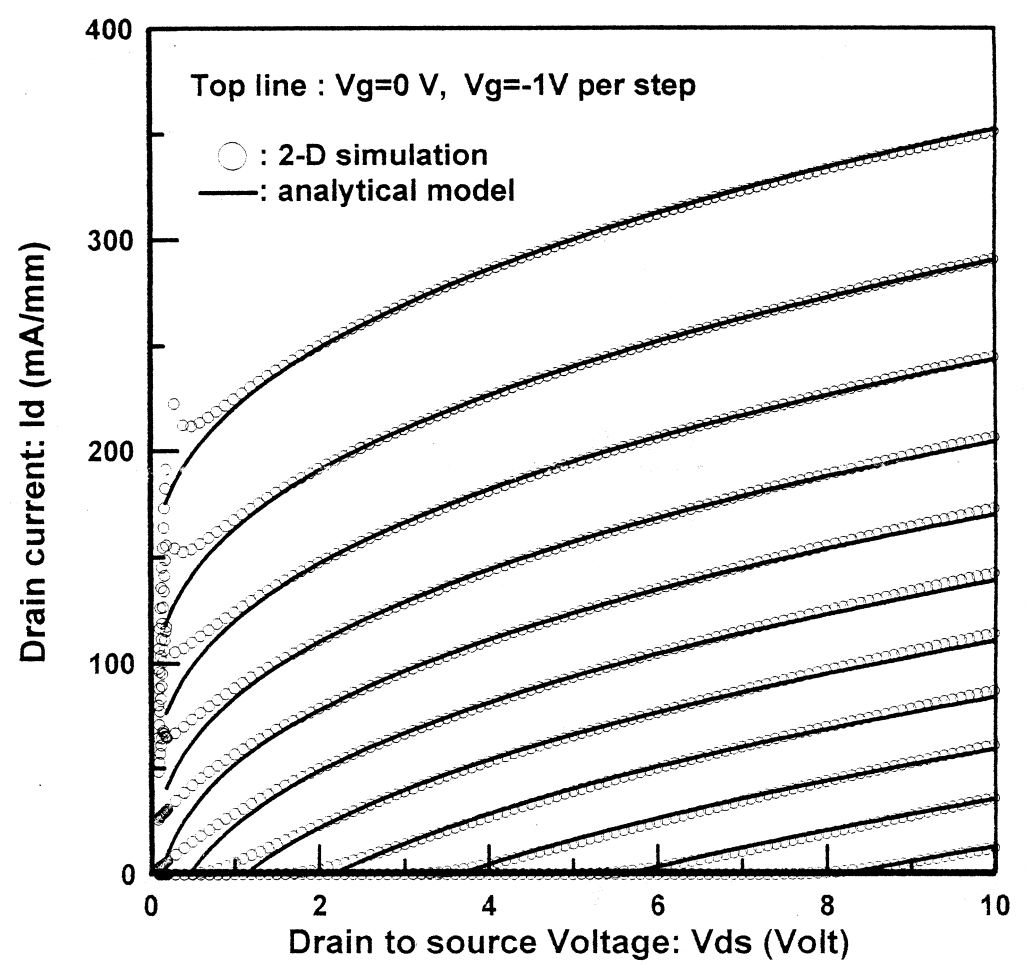

Fig. 9. $I-V$ characteristics of the $\mathrm{V}$-groove MESFET's with undoped $\mathrm{Al}_{0.3} \mathrm{Ga}_{0.7} \mathrm{As}$ and the fitting results of the DICA analytical model.

In the beginning, we knew that the electron accumulation of the dipole region was formed just under the tip of the V-groove gate. Like Fig. 4, we can sketch the electron distribution at $V_{\mathrm{d}}=1,3,5$ and $10 \mathrm{~V}$ and $V_{\mathrm{g}}=0$ along the cut line from the V-groove's tip to the substrate's bottom as in Fig. 11(a). We can see clearly that some electrons were pushed out of the channel into the substrate and that the concentration of the pushed electron became larger as $V_{\mathrm{d}}$ increased. (Here $y=0$ denotes the bottom of substrate; $y=0.8$, the junction between channel and substrate and $y=1.0$, the V-groove's tip, as shown in Fig. 1.) Also, although the peak electron concentration in the channel seems to increase with $V_{\mathrm{d}}$, we will find that the total number of the electrons in the channel almost maintains the same value. The effect of electron pushout not only caused the leakage current in the substrate but also prevented the DICA effect in the channel, because the electron cannot accumulate too much in the channel (if it accumulates too much, it will be pushed into the substrate).

Similarly, in the device with undoped AlGaAs buffer, Fig. 11(b) is the electron concentration under the $\mathrm{V}$-groove tip at $V_{\mathrm{g}}=0$ and $V_{\mathrm{d}}=1,3,5$ and $10 \mathrm{~V}$. In Fig. 11(b), we can see that no electrons were pushed out into the AlGaAs and that the electron concen- tration is increasing with $V_{\mathrm{d}}$. The DICA effect is significant. This is because the conduction band discontinuity $\left(\Delta E_{\mathrm{c}}\right)$ of the $\mathrm{AlGaAs} / \mathrm{GaAs}$ heterojunction prevents the electrons being pushed out into the substrate and as a consequence it accumulates in the channel and causes the DICA effect. A very high peak of electron concentration was found at the junction. This is because of the band bending of $\mathrm{AlGaAs} / \mathrm{GaAs}$ heterojunction as shown in Fig. 12.

One very interesting thing is that the short channel effect in the device with the p-type substrate is just between the above two cases. We can see in Fig. 11(c) that the DICA effect appears at small $V_{\mathrm{d}}$ and the substrate leakage effect appears at large $V_{\mathrm{d}}$. These reveal that the p-type buffer can prevent a certain amount of electron from being pushed out into the substrate but when the electron accumulated too much, the electrons still can overcome the potential barrier, inject into the substrate and hence cause the leakage current. So, the short channel effect of the device with p-type substrate is a combination of the substrate leakage and the DICA effect.

Finally, one thing which has to be pointed out is that the channel current is proportional to the total carrier accumulated under the V-groove tip in the channel. The total carrier can be calculated by inte- 

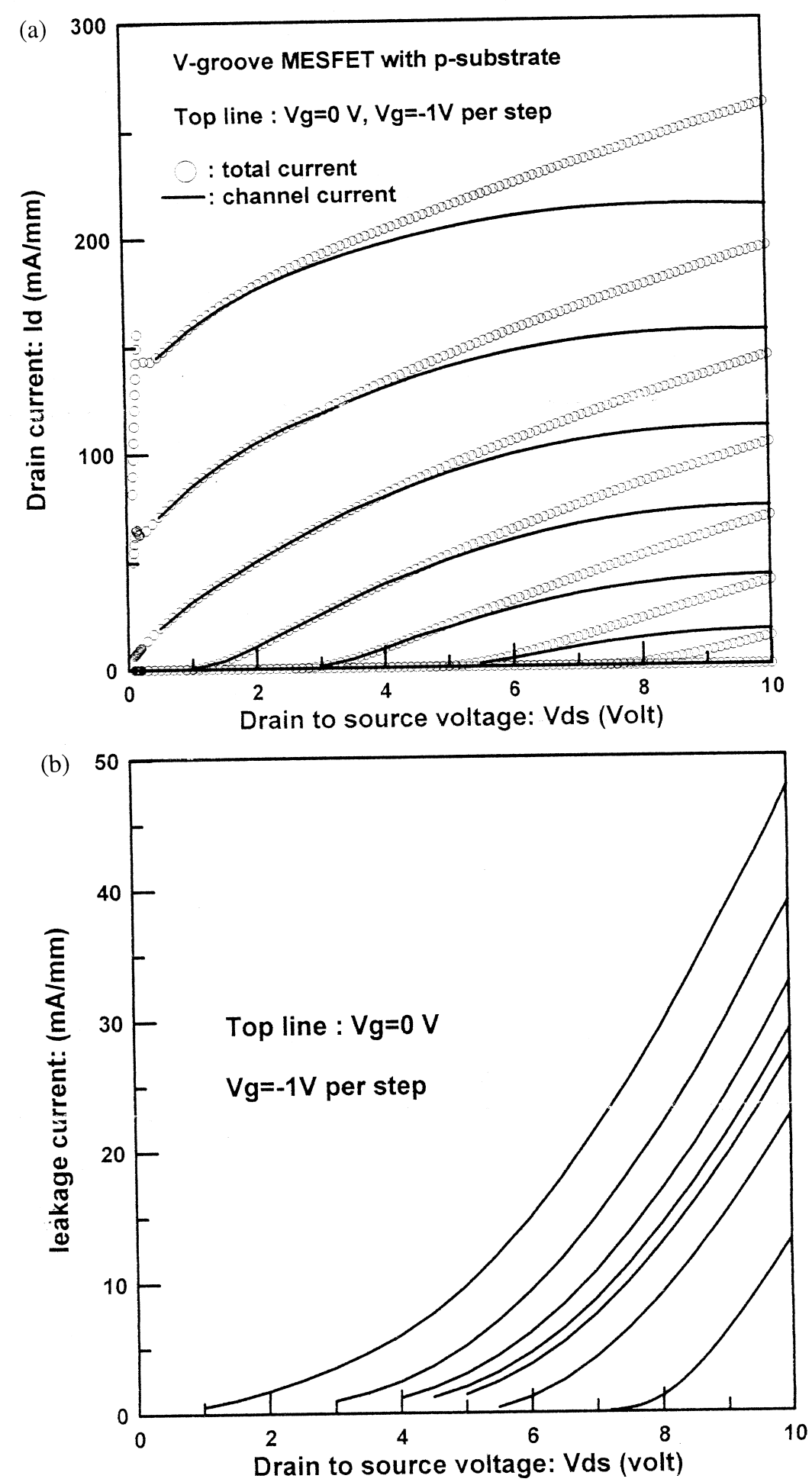

Fig. 10. (a) Channel current and (b) substrate leakage current of the V-groove MESFET with $10^{16} / \mathrm{cm}^{3}$ p-type GaAs substrate. 

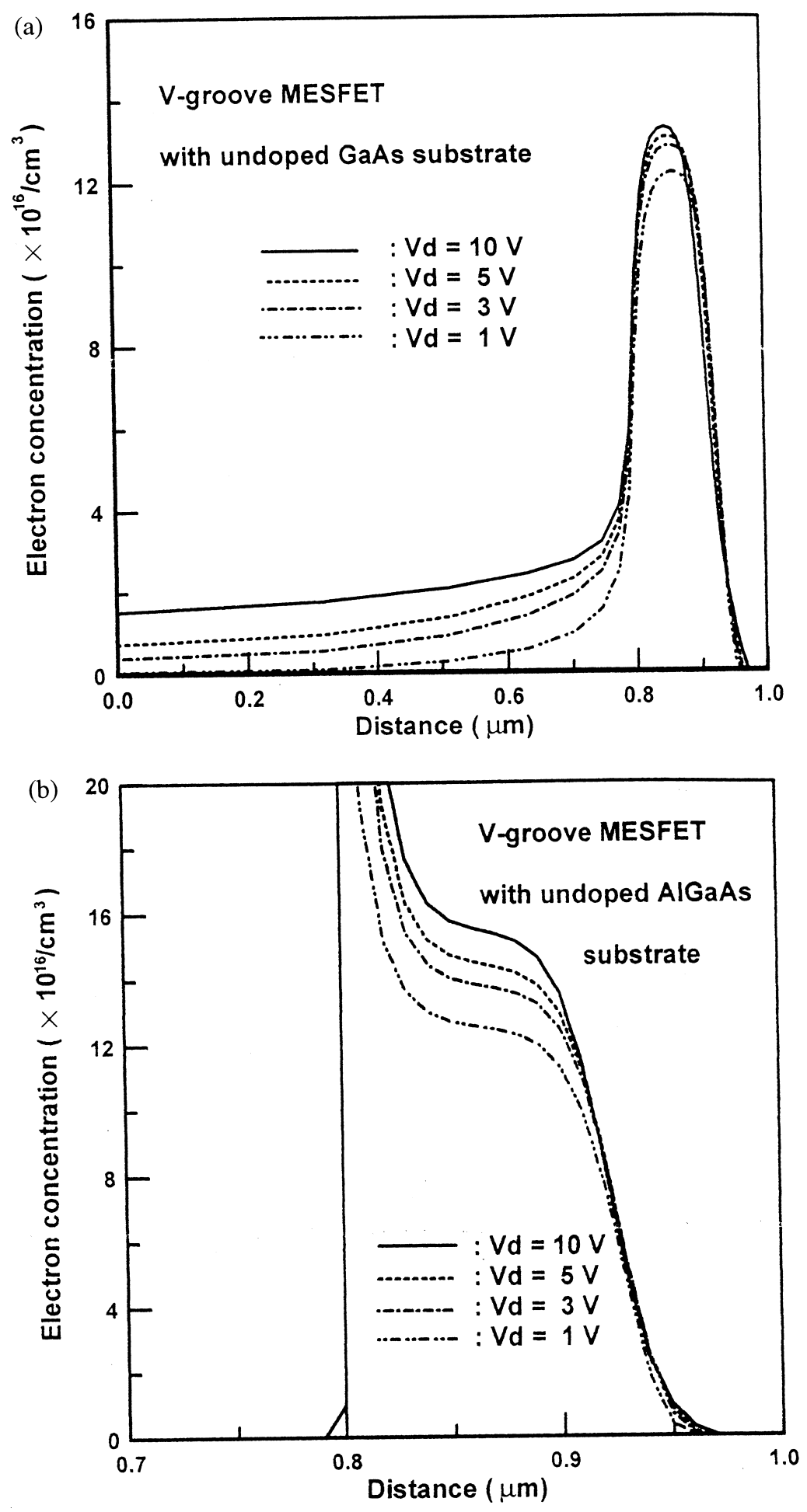

Fig. 11. Electron concentration under the V-groove tip of the V-groove MESFET with (a) undoped GaAs substrate (b) with undoped $\mathrm{Al}_{0.3} \mathrm{Ga}_{0.7} \mathrm{As}$ substrate and (c) $10^{16} / \mathrm{cm}^{3}$ p-type $\mathrm{GaAs}$ substrate $\left(V_{\mathrm{d}}=1,3,5,10 \mathrm{~V} ; V_{\mathrm{g}}=0 \mathrm{~V}\right)$. 


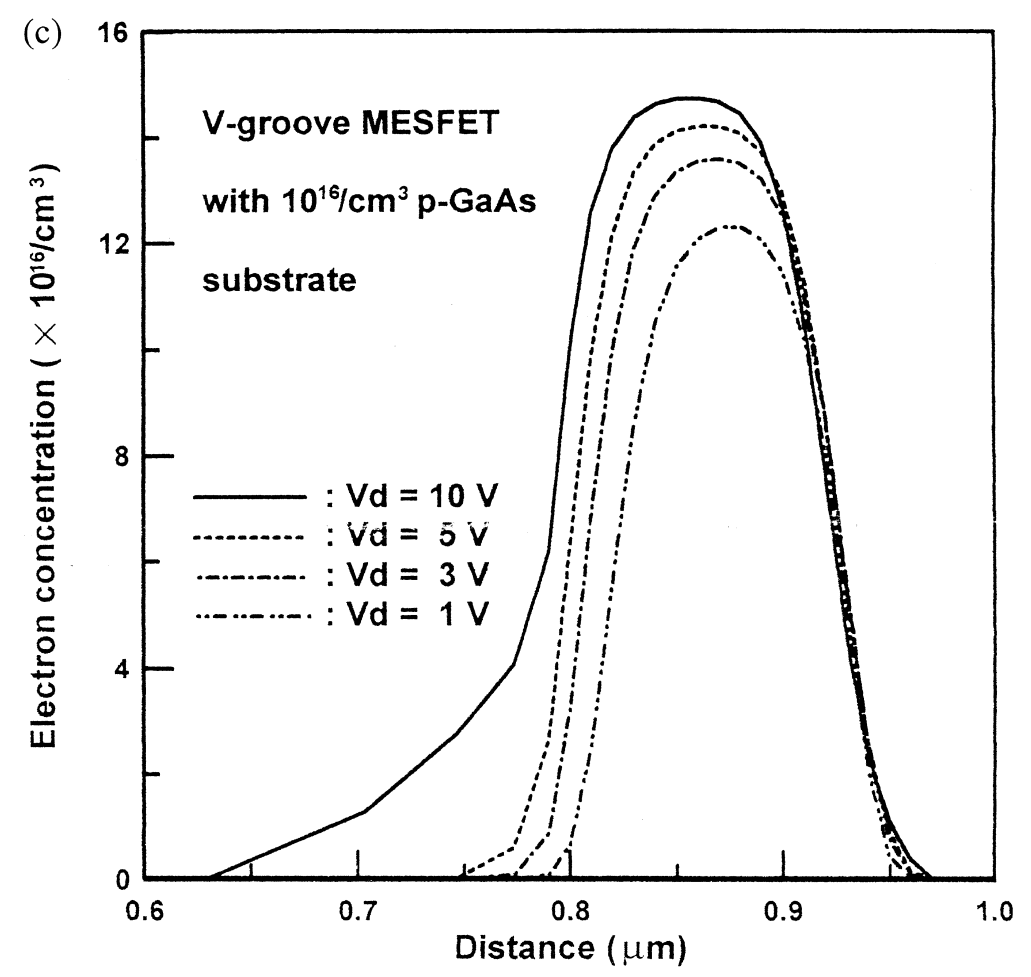

Fig. 11 (continued)

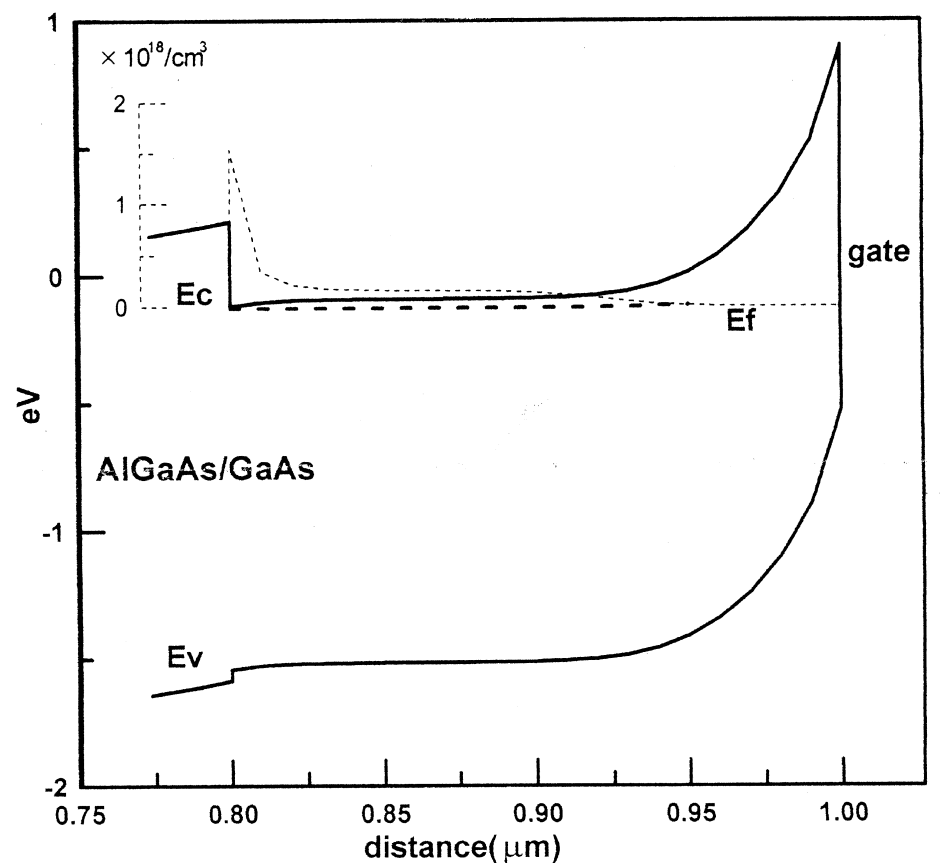

Fig. 12. Band diagram and the electron concentration under the tip of the V-groove MESFET with undoped GaAs, substrate $\left(V_{\mathrm{d}}=1 \mathrm{~V} ; V_{\mathrm{g}}=0 \mathrm{~V}\right)$. 

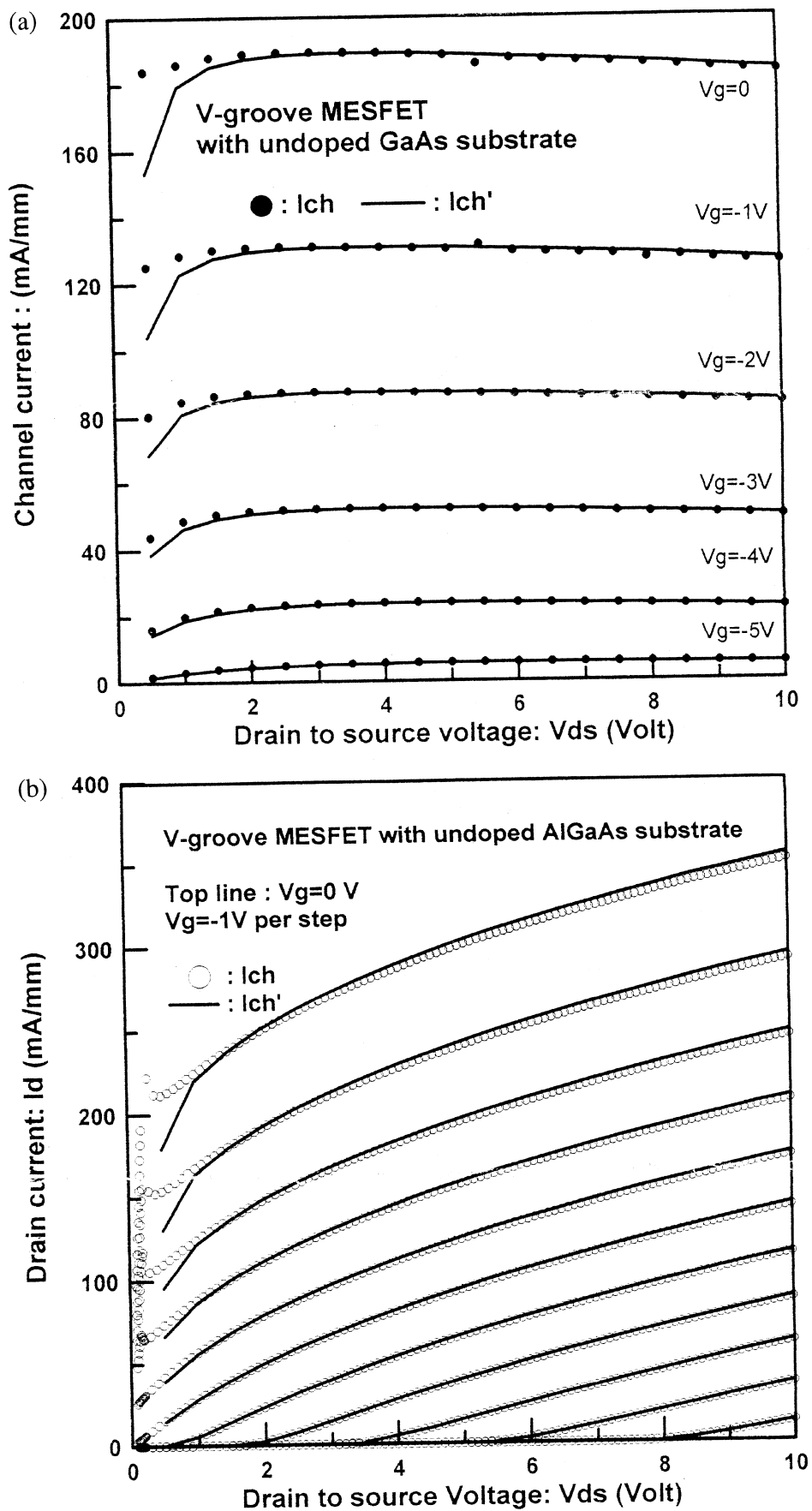

Fig. 13. Comparison of the simulated channel current $\left(I_{\mathrm{ch}}\right)$ and the current calculated by the electron saturation velocity $\left(I_{\mathrm{ch}}{ }^{\prime}\right)$ of the V-groove MESFET's with (a) undoped GaAs, (b) undoped $\mathrm{Al}_{0.3} \mathrm{Ga}_{0.7} \mathrm{As}$ and (c) $10^{16} / \mathrm{cm}^{3}$ p-type GaAs substrate. 


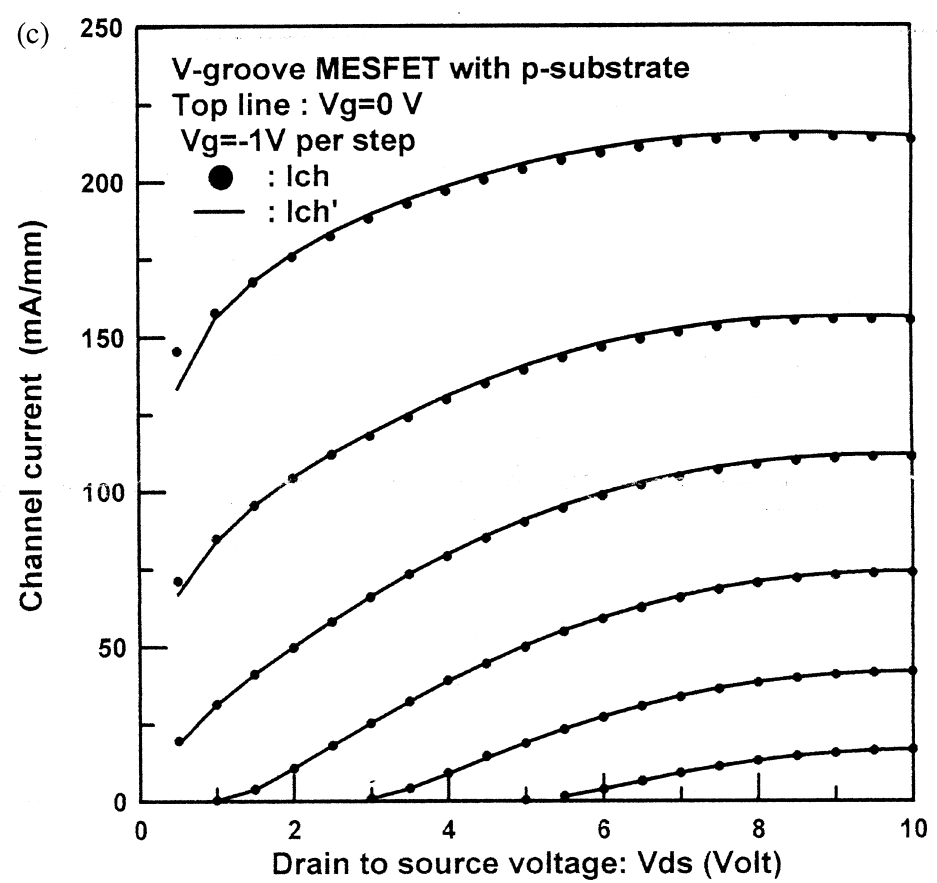

Fig. 13 (continued)

grating the total electron under the $\mathrm{V}$-groove tip in the channel. Because the electric field under the tip is the strongest, the carrier here must drift by the saturation velocity. So we can calculate the channel current as follows:

$$
I_{\mathrm{ch}}^{\prime}=v_{\mathrm{s}} \times \int_{\text {channel }} n \times d l(\mathrm{~mA} / \mathrm{mm})
$$

where $n$ represents the electron concentration and $v_{\mathrm{s}}$, the saturation velocity.

We plotted $I_{\mathrm{ch}}{ }^{\prime}$ and the simulated channel current $I_{\mathrm{ch}}$ and found that they match very well except at small $V_{\mathrm{d}}$, as shown in Fig. 13(a), (b) and (c). It is reasonable because at small $V_{\mathrm{d}}$, electrons have higher velocity than the saturation velocity. Therefore, the DICA effect that we presented is more convincing.

\section{Conclusion}

With the help of 2D simulation, the DICA effect in the V-groove gate GaAs MESFET was found. The DICA effect could be described by equations based on the simple velocity saturation model. Also, the mechanisms of the short channel effect of different substrates in the GaAs V-groove gate MESFET's were identified by two-dimensional simulation. In the device with undoped GaAs substrate, the mechanism of the short-channel effect was the current injection into the substrate. However, in that with the undoped AlGaAs substrate, it was the DICA effect. As for the device with the p-doped GaAs substrate, both the current injection and the DICA were found.

These results reveal that the substrate leakage and the DICA effect are two mutual competitive mechanisms, i.e. if we tried to eliminate one mechanism then the other mechanism will appear. The reason is that the electrons are either pushed into the substrate to cause the substrate leakage or accumulate in the channel to cause the DICA effect. In consequence, the short channel effect is unavoidable in the $\mathrm{V}$-groove gate MESFET at all.

\section{Acknowledgements}

This work is supported by the National Science Council of ROC under contract No. NSC84-2512-S002-008.

\section{References}

[1] Kennedy DP, O'Brien RR. IBM J Res Dev 1970;14:95.

[2] Reiser M. Elec Lett 1970;6:493.

[3] Yamaguchi K, Asai S, Kodera H. IEEE Trans Electron Devices 1976;23:1283. 
[4] Kohn E. Elec Lett 1975;11:160.

[5] Wang YJ, Lu SS. Elec Lett 1997;33:538.

[6] Eastman LF, Shur MS. IEEE Trans Electron Devices 1979;26:1359.

[7] Iida S, Ito K. J Electrochem Soc 1971;118:768.

[8] Butcher PN, Faweett W. Phys Lett 1966;21:489.
[9] Ruch JG, Kino GS. Appl Phys Lett 1967;10:40.

[10] Copeland JA. J Appl Phys 1967;38:3096.

[11] Thim HW. J Appl Phys 1968;39:3897.

[12] Barnes JJ, Lomax RJ. Elec Lett 1974;10:341.

[13] Barnes JJ, Lomax RJ, Haddad GI. IEEE Trans Elec Dev 1976;21:1042. 